Europhys. Lett., 54 (1), pp. 105-111 (2001)

\title{
Electron-electron interaction effects in magnetic layered structures
}

\author{
V. K. Dugaev ${ }^{1}\left(^{*}\right)$ and J. BARnAś ${ }^{2}$ \\ 1 Chernovtsy Department of the Institute of Materials Science Problems \\ Vilde 5, 58001 Chernovtsy, Ukraine \\ 2 Department of Physics, A. M. University \\ ul. Umultowska 85, 61-614 Poznań, Poland \\ and Institute of Molecular Physics, Polish Academy of Sciences \\ ul. M. Smoluchowskiego 17, 60-179 Poznań, Poland
}

(received 9 October 2000; accepted in final form 26 January 2001)

PACS. 75.70.-i - Magnetic properties of thin films, surfaces and interfaces.

PACS. 75.70.Cn - Interfacial magnetic properties (multilayers).

PACS. 73.20.Fz - Weak or Anderson localization.

\begin{abstract}
We consider some peculiarities of the logarithmic electron-electron interaction corrections to conductivity, which can arise in magnetic quantum wells due to spin-splitting of energy levels. The matrix elements of Coulomb interaction include different screening contributions from spin-up and spin-down electrons, which can lead to anomalous behavior of the interaction constant when the width of a spin quantum well is changed. Our results explain qualitatively the physical origin of the recently observed anomalous behavior of the logarithmic term in electrical resistivity of $\mathrm{Co} / \mathrm{Cr} / \mathrm{Ag} /$ Co layered structures (Aliev F. G. et al., Phys. Rev. Lett., 78 (1997) 134).
\end{abstract}

There has been a great interest recently in confined structures with magnetic and nonmagnetic materials, like metallic magnetic multilayers or magnetic tunnel junctions [1-4]. An important feature of such systems is the existence of spin quantum wells (SQWs), i.e., different quantum wells for electrons with opposite spin orientations. Although a lot of work has been done on transport properties of magnetic layered structures, the low-temperature regime, where quantum corrections due to weak localization and electron-electron interaction are important [5-7], is still not well understood [1,8-10]. The main issue is related to an internal magnetic field which may suppress the localization corrections. However, nonvanishing localization corrections were observed, e.g., in percolating Ni films [10]. On the other hand, the available results on low-temperature transport properties of epitaxial iron films [9] can be explained by both localization and interaction corrections. As is well known, the electronelectron interaction corrections are weakly sensitive to magnetic field and therefore can be expected to play a significant role in magnetic materials [11].

$\left(^{*}\right)$ Present address: Max-Planck-Institut für Mikrostrukturphysik - Weinberg 2, D-06120 Halle, Germany. 


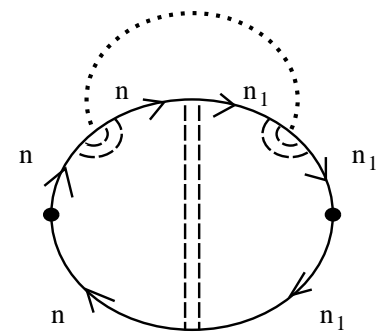

Fig. 1 - Exchange corrections to conductivity.

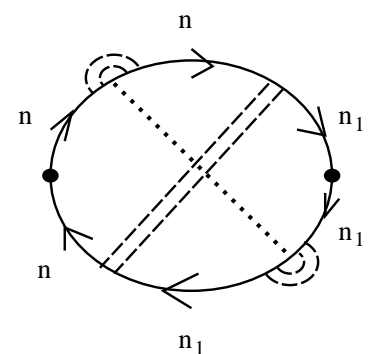

In ref. [1] the temperature dependence of the electrical resistivity of ultra-thin $\mathrm{Co} / \mathrm{Cr} / \mathrm{Ag} / \mathrm{Co}$ structures was measured down to $30 \mathrm{mK}$. The thickness of the Co layers was about $45 \AA$, whereas the thickness $L_{0}$ of the $(\mathrm{Cr} / \mathrm{Ag})$ bilayer was monotonously changed at a constant ratio of the $\mathrm{Cr}$ and $\mathrm{Ag}$ thicknesses. It was shown that the low-temperature logarithmic term in resistivity can be described as the quantum correction arising from electron-electron interaction in a two-dimensional (2D) case. The most important feature of the logarithmic term was its anomalous dependence on $L_{0}$. More specifically, a few large and small narrow peaks were found, respectively, at $L_{0} \approx 10,15,20,25$ and $30 \AA$. However, the physical origin of those peaks is still unclear. In this letter we show that the possible origin of the peaks follows from some peculiarities in electron screening, which can arise in SQWs due to spin-splitting of the energy levels.

We consider a SQW in the strong quantization limit, i.e., when the discrete energy levels due to quantization of perpendicular motion are well separated, $\Delta \varepsilon \gg T(\Delta \varepsilon$ is the level separation and we put the Boltzmann constant $\left.k_{\mathrm{B}}=1\right)$. We will distinguish the two sets of energy levels with the indices $n$ (or $n_{1}$ ) for spin-up ( $\uparrow$ ) and $m$ (or $m_{1}$ ) for spin-down $(\downarrow)$ electrons, i.e., $\varepsilon_{n}$ and $\varepsilon_{m}$. For SQWs these two sets of energy levels are different and our main objective is to analyse how this difference affects the quantum correction to conductivity due to electron-electron interaction. In the simplest case the two quantum wells are approximated by hard-wall ones with different well widths, $L_{\uparrow}$ and $L_{\downarrow}$, for electrons with opposite spin orientations.

Further considerations will be carried out on the following assumptions. First, we assume the inter-subband scattering rate to be roughly equal to the intra-subband one. This seems to be reasonable for a short-range scattering potential of structural imperfections and means that we should consider diagrams with all $n \rightarrow n_{1}$ (and $m \rightarrow m_{1}$ ) transitions associated with impurity or defect scattering. Second, we neglect spin-flip scattering (like $n \rightarrow m$ transitions) which is usually associated with spin-orbit interaction or scattering from magnetic impurities. The third condition we adopt concerns size quantization of Diffusons. If we assume $\Delta \varepsilon_{n}, \Delta \varepsilon_{m}>1 / \tau_{\uparrow(\downarrow)}$ (where $\tau_{\uparrow(\downarrow)}$ is the spin-dependent elastic relaxation time, and we put $\hbar=1$ ), only diagrams with equal $n$ (or $m$ ) for the particle and hole lines should be taken into account. This leads to quasi-two-dimensionality in all formulas for the quantum corrections.

The relevant diagrams for exchange corrections to conductivity in SQWs are shown in fig. 1 (and similar ones with $n$ replaced by $m$ ). The dot-line corresponds there to the matrix elements of the Coulomb interaction and the double-dashed lines stand for the Diffusons. In these diagrams, small values of the frequency $\omega$ and momentum transferred through the interaction line are essential; $\omega, D_{\uparrow(\downarrow)} q^{2} \ll 1 / \tau_{\uparrow(\downarrow)}$ (here, $D_{\uparrow(\downarrow)}$ is the spin-dependent diffusion 


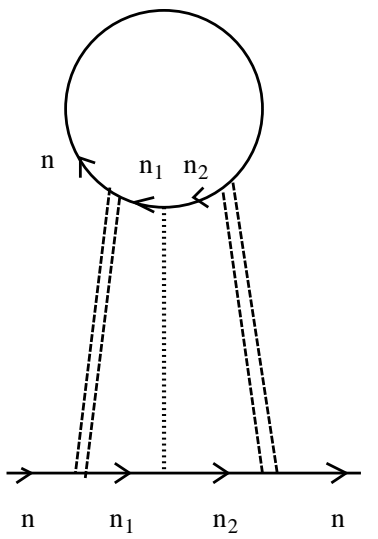

Fig. 2

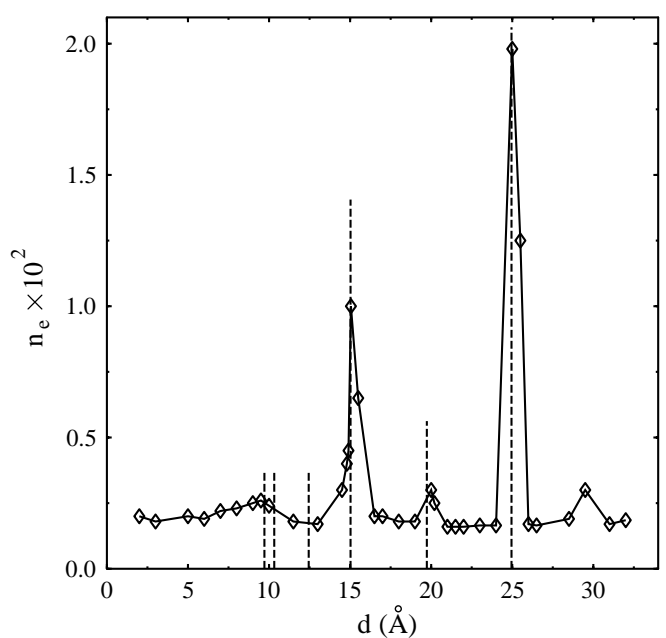

Fig. 3

Fig. 2 - The Hartree diagram.

Fig. 3 - Position of the peaks in $\lambda$ (vertical dashed lines), when the thickness of the $\mathrm{Cr} / \mathrm{Ag}$ bilayer is changed. Experimental data obtained in ref. [1] are also reproduced.

constant, $q$ is a two-dimensional wave vector and to avoid cumbersome pictures we do not indicate $\omega$ and $q$ on the diagrams). The corresponding contribution to electrical conductivity is given by

$$
\Delta \sigma_{\mathrm{ex}}=\frac{e^{2}}{2 \pi^{2}}\left[\frac{\rho_{\uparrow}}{\rho_{\uparrow}+\rho_{\downarrow}} \ln \left(T \tau_{\uparrow}\right)+\frac{\rho_{\downarrow}}{\rho_{\uparrow}+\rho_{\downarrow}} \ln \left(T \tau_{\downarrow}\right)\right],
$$

where $\rho_{\uparrow}=\rho_{0} N_{\uparrow}$ and $\rho_{\downarrow}=\rho_{0} N_{\downarrow}$ are the densities of states at the Fermi level for spin-up and spin-down electrons, respectively. Here, $N_{\uparrow}$ and $N_{\downarrow}$ denote the corresponding numbers of discrete energy levels under the Fermi level (numbers of $2 \mathrm{D}$ subbands) and $\rho_{0}$ is the $2 \mathrm{D}$ density of states, $\rho_{0}=m^{\star} / 2 \pi$ (with $m^{\star}$ being the effective electron mass). When deriving eq. (1) we took into account screening of the electron-electron interaction in SQWs, which at small values of the transferred energy and momentum gives

$$
V_{n n}(q, \omega)=V_{m m}(q, \omega)=2 \pi e^{2}\left[q+2 \pi e^{2}\left(\rho_{\uparrow} \frac{D_{\uparrow} q^{2}}{-i \omega+D_{\uparrow} q^{2}}+\rho_{\downarrow} \frac{D_{\downarrow} q^{2}}{-i \omega+D_{\downarrow} q^{2}}\right)\right]^{-1},
$$

where the diffusion constants $D_{\uparrow}$ and $D_{\downarrow}$ are given, respectively, by $D_{\uparrow}=\left(1 / N_{\uparrow}\right) \sum_{n} v_{\mathrm{F} n}^{2} \tau_{\uparrow} / 2$ and $D_{\downarrow}=\left(1 / N_{\downarrow}\right) \sum_{m} v_{\mathrm{F} m}^{2} \tau_{\downarrow} / 2$. Here, $v_{\mathrm{F} n(m)}$ is the Fermi velocity in the $n(m)$-th subband, and the unscreened Coulomb interaction becomes purely two-dimensional at sufficiently small values of $q, V_{n n}^{(0)}(q)=2 \pi e^{2} / q$ at $q \ll 1 / L_{\uparrow}$.

Let us consider now the Hartree-type contribution to the quantum correction, which is the main point of our work. As is well known [5-7], the Hartree-type contribution to conductivity can be calculated directly from the corrections to the density of electron states. Among the diagrams describing these corrections there are diagrams with Diffusons and Cooperons. However, we can omit diagrams with Cooperons, either when $T \tau_{\varphi \uparrow(\downarrow)} \ll 1$ (where $\tau_{\varphi \uparrow(\downarrow)}$ is the spin-dependent phase coherence time) or when we assume strong internal magnetization. 
The relevant diagram describing correction to the density of states is shown in fig. 2. It contains the matrix elements of Coulomb interaction for transitions between different subbands (without spin reversal) with the transferred frequency $\omega=0$ and 2D momentum $q \sim k_{\mathrm{F}}$ (with $k_{\mathrm{F}}$ being the Fermi momentum in the absence of size quantization). This is a renormalized interaction which takes into account screening by free carriers of both spin orientations. Generally, the dependence of renormalized interaction on $\omega$ is related to the dynamical character of the screening. The contribution of the Hartree diagrams to conductivity is similar to the conventional $2 \mathrm{D}$ theory [5-7] and is given by

$$
\Delta \sigma_{\mathrm{Har}}=-\frac{e^{2}}{4 \pi^{2}} \cdot \frac{1}{2}\left[F_{\uparrow} \ln \left(T \tau_{\uparrow}\right)+F_{\downarrow} \ln \left(T \tau_{\downarrow}\right)\right]
$$

where

$$
F_{\uparrow}=\frac{\left\langle V_{n n_{1}}(q, 0)\right\rangle}{V_{n n}(0,0)}
$$

and

$$
F_{\downarrow}=\frac{\left\langle V_{m m_{1}}(q, 0)\right\rangle}{V_{m m}(0,0)}
$$

are the coupling constants. Here, $V_{n n}(0,0)=V_{m m}(0,0)$ are the matrix elements of renormalized interaction (screened by electrons of both spin orientations, eq. (2)). The averaging of the matrix elements $V_{n, n_{1}}(q, 0)$ and $V_{m, m_{1}}(q, 0)$ is here over all allowed transitions at the Fermi surface. The latter matrix elements are crucial for our analysis, as will be described later.

The sign of the Hartree contribution is opposite to the sign of the exchange term. As for the additional factor, we adopt here the results of extensive discussion on right counting of states within triplet interaction channel $[6,7,12]$. In the absence of different quantization for spin-up and spin-down electrons, we should get the factor $3 / 2$ related with all triplet states. In our case, however, we take into account only states with $S_{z}= \pm 1$.

The matrix element of the unscreened Coulomb interaction with a basis set of wave functions $\psi_{n}(z)$ describing quantized states can be written as

$$
V_{n n_{1}}^{(0)}(q)=\int \frac{\mathrm{d} p}{2 \pi} \frac{4 \pi e^{2}}{q^{2}+p^{2}}\left|I_{n n_{1}}(p)\right|^{2}
$$

where

$$
I_{n n_{1}}(p)=\int \mathrm{d} z e^{i p z} \psi_{n}^{*}(z) \psi_{n_{1}}(z)
$$

To simplify further considerations we assume $\psi_{n}(z)$ in the form of eigenfunctions of a rectangular hard-wall quantum well of width $L_{\uparrow}$ for spin $s=\uparrow$ and width $L_{\downarrow}$ for spin $s=\downarrow$, i.e.,

$$
\psi_{n}(z)=\sqrt{\frac{2}{L_{\uparrow}}} \sin \frac{n \pi z}{L_{\uparrow}}
$$

and a similar formula for the opposite spin orientation. In that case, for $n\left(n_{1}\right) \sim N_{\uparrow} \sim k_{\mathrm{F}}^{2} L_{\uparrow}^{2}$, $n \neq n_{1}$, and $q \sim k_{\mathrm{F}} \ll 2 \pi n / L_{\uparrow}, 2 \pi n_{1} / L_{\uparrow}$, one finds the following approximate expression:

$$
\begin{aligned}
\left|I_{n n_{1}}(p)\right| & \simeq \frac{1}{2 L_{\uparrow}}\left[\delta\left(p-\kappa_{n}+\kappa_{n_{1}}\right)+\delta\left(p+\kappa_{n}-\kappa_{n_{1}}\right)+\right. \\
& \left.+\delta\left(p-\kappa_{n}-\kappa_{n_{1}}\right)+\delta\left(p+\kappa_{n}+\kappa_{n_{1}}\right)\right]
\end{aligned}
$$


where $\kappa_{n}=n \pi / L_{\uparrow}$ and we have neglected the terms $\sim\left(p L_{\uparrow}\right)^{-1}$, which are small due to the oscillating integrand in eq. (7) for $p \sim k_{\mathrm{F}} \gg 1 / L_{\uparrow}$. Thus, in the matrix elements (6) the momentum $p=\kappa_{n} \pm \kappa_{n_{1}}$ is transferred through the interaction line.

When considering the screening diagrams in the framework of RPA approximation, we should take into account matrix elements linking electrons with the same or different spin orientations. Due to eq. (9), the latter matrix elements are nonzero provided $\kappa_{n} \pm \kappa_{n_{1}}=$ $\kappa_{m} \pm \kappa_{m_{1}}$. This can happen only for some specific values of $L_{\uparrow}$ and $L_{\downarrow}$, i.e., when $L_{\uparrow}=l L_{\downarrow}$, where $l$ is an integer number, $l \in \mathcal{I}$ (for $L_{\uparrow}>L_{\downarrow}$ ). Thus, the contribution of polarization diagrams gives the inverse screening length $\xi_{\uparrow(\downarrow)}=4 \pi e^{2} \rho_{\uparrow(\downarrow)}$, except for some special values of $L_{\uparrow}$ and $L_{\downarrow}$ (referred to as special points), when $L_{\uparrow} / L_{\downarrow}$ (or $L_{\downarrow} / L_{\uparrow}$ ) is an integer number. In that case we should put the total density of states $\rho=\rho_{\uparrow}+\rho_{\downarrow}$ instead of $\rho_{\uparrow}$ and $\rho_{\downarrow}$. We can thus write $\xi_{\uparrow(\downarrow)} \simeq 4 \pi e^{2} \rho_{\uparrow(\downarrow)}$ everywhere except for special points, where $\xi_{\uparrow(\downarrow)} \simeq \xi=4 \pi e^{2} \rho$. Note that the screening in $V_{n n}(0,0)$ always contains the total density of states $\rho$.

Thus, we can write

$$
V_{n n_{1}}(q, 0)=\frac{4 \pi e^{2}}{L_{\uparrow}\left[q^{2}+\left(\kappa_{n}-\kappa_{n_{1}}\right)^{2}\right]+\xi_{\uparrow}}
$$

and, in accordance with eq. (2),

$$
V_{n n}(0,0)=\frac{4 \pi e^{2}}{\xi}
$$

Taking this into account we obtain

$$
F_{\uparrow}=\frac{1}{N_{\uparrow}^{2}} \sum_{n n_{1}} \frac{\xi}{\left[\left(2 k_{F}^{2} L_{\uparrow}-2 \kappa_{n} \kappa_{n_{1}} L_{\uparrow}+\xi_{\uparrow}\right)^{2}-4\left(k_{F}^{2}-\kappa_{n}^{2}\right)\left(k_{F}^{2}-\kappa_{n_{1}}^{2}\right) L_{\uparrow}^{2}\right]^{1 / 2}},
$$

where the summation is over all states under the Fermi level. A similar formula holds also for $F_{\downarrow}$. The averaging in eq. (4) (or (5)) is over transitions at the Fermi surface, and therefore also includes the range of small values of $q$ (particularly for $n=n_{1}$ ). The relative contribution due to the small- $q$ range is however negligible, so we use formula (10) for the whole range of $q$.

The total contribution from electron-electron interaction is the sum of exchange and Hartree diagrams and can be written as

$$
\Delta \sigma=\frac{e^{2}}{4 \pi^{2}} \lambda \ln (T \tau)
$$

where $\tau=\left(\tau_{\uparrow}+\tau_{\downarrow}\right) / 2$, and

$$
\lambda=2-\left(F_{\uparrow}+F_{\downarrow}\right) / 2 .
$$

In the case of strong screening one has to include higher-order terms in the perturbation expansion, especially the ladder Hartree diagrams $[6,7]$. In a $2 \mathrm{D}$ case the conductivity correction is still given by eqs. (13) and (14), but with $F_{\uparrow}$ and $F_{\downarrow}$ replaced by renormalized constants, $\tilde{F}_{\uparrow}$ and $\tilde{F}_{\downarrow}$, which are related with $F_{\uparrow}$ and $F_{\downarrow}$ according to the formula

$$
\tilde{F}_{\uparrow(\downarrow)}=8\left(1+F_{\uparrow(\downarrow)} / 2\right) \ln \left(1+F_{\uparrow(\downarrow)} / 2\right) / F_{\uparrow(\downarrow)}-4 .
$$

In the case of very strong screening, $\xi_{\uparrow}, \xi_{\downarrow} \gg k_{\mathrm{F}}$, we obtain $F_{\uparrow} \simeq \xi / \xi_{\uparrow}$ and $F_{\downarrow} \simeq \xi / \xi_{\downarrow}$, and consequently $\tilde{F}_{\uparrow(\downarrow)} \simeq 8\left(1+\rho / 2 \rho_{\uparrow(\downarrow)}\right)\left(\rho_{\uparrow(\downarrow)} / \rho\right) \ln \left(1+\rho / 2 \rho_{\uparrow(\downarrow)}\right)-4$ for all values of $L_{\uparrow}$ and $L_{\downarrow}$, except for special points where $\tilde{F}_{\uparrow(\downarrow)} \simeq 0$.87. Thus, when for instance $\rho_{\downarrow} / \rho_{\uparrow}=3$, we obtain 
$\lambda=0.15$, except for special points where $\lambda \simeq 1.13$. If the screening is weak, one still can expect an increase in the magnitude of $\lambda$ at special points, which can be calculated numerically by taking into account eqs. (10) to (14).

Thus, we have shown that one can expect nonmonotonous behavior of the $\lambda$-factor describing electron-electron interaction corrections to conductivity with increasing thickness of the structure. In the simple case, when SQW can be approximated by a hard-wall quantum well with thickness $L_{\uparrow}$ for spin-up and $L_{\downarrow}$ for spin-down electrons, the factor $\lambda$ can be significantly enhanced for integer values of $L_{\uparrow} / L_{\downarrow}$ (or $L_{\uparrow} / L_{\downarrow}$ ). In real systems, however, SQWs are more complex and the difference in screening lengths at the special points and between the points can be smaller than that predicted by the simple model considered here.

The presence of large narrow peaks in the dependence of the coupling constant $\lambda$ on the width of the $\mathrm{Ag}$ layer observed in $\mathrm{Co} / \mathrm{Cr} / \mathrm{Ag} / \mathrm{Co}$ structures [1] is consistent with our considerations. A rough estimation of the magnitude and width of peaks is also consistent with experimental observations. As follows from eq. (6), the width of $\delta$-peaks in eq. (9) can be estimated as $\Delta \ll 1 / k_{\mathrm{F}}$ and for $\varepsilon_{\mathrm{F}} \simeq 8 \mathrm{eV}$ this gives $\Delta \ll 3 \AA$. The magnitude of peaks can be estimated as $\sim 1$. From the reasons discussed above, the simplified model of a SQW cannot give correct positions of the peaks. But we hope that it offers a mechanism which may be responsible for the experimental observations.

We can now estimate the positions of the peaks in $\lambda$ within the simplified model, by taking into account the relevant band structure parameters $[13,14]$ and the parameters corresponding to the experimental situation of ref. [1]. The spin-down quantum well corresponds then to the $\mathrm{Ag}$ sublayer, while the spin-up quantum well corresponds to the $\mathrm{Ag} / \mathrm{Co}$ bilayer. According to the experimental conditions of ref. [1], $L_{\downarrow} \simeq(1.35 / 2.35) d$ (where $d$ is the thickness of the $\mathrm{Cr} / \mathrm{Ag}$ bilayer), and $L_{\uparrow} / L_{\downarrow} \simeq 1+75 / d$. The condition $L_{\uparrow} / L_{\downarrow}=l$ (with $l$ integer) then gives $d \simeq 75 /(l-1)$. Values of $d$ obtained from this condition for $l$ ranging from $l=4$ to $l=9$ are presented in fig. 3 as dashed vertical lines, where also the experimental data are reproduced from ref. [1]. Despite the simplifications, agreement between experimental and theoretical positions of the peaks is quite satisfactory.

In conclusion, we have proposed a mechanism leading to large peaks in the logarithmic correction to conductivity due to electron-electron interaction in magnetic layered structures. The peaks occur in the thickness dependence of the factor $\lambda$ and originate from some peculiarities in electron screening, which are due to spin-splitting of the electron subbands in SQWs. Our results may explain the origin of a recently observed enhancement of the logarithmic term in resistivity of $\mathrm{Co} / \mathrm{Cr} / \mathrm{Ag} / \mathrm{Co}$ structures, which was found at certain values of the $\mathrm{Cr} / \mathrm{Ag}$ bilayer thicknesses [1]. The key point of the model is the existence of SQWs, which result from the Stoner splitting of the electron bands in transition metals. Thus, similar effects can also be observed in simpler structures, like, for instance, in $\mathrm{Co} / \mathrm{Ag} / \mathrm{Co}$ trilayers or $\mathrm{Co} / \mathrm{Ag}$ bilayers. The interlayer exchange coupling is not a necessary condition for the effects - although it may have an influence on the peak positions. This is because the position of the peaks may depend on the magnetic configuration of the system.

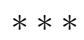

This work was supported by the Polish Committee for Scientific Research under Grant 8T11F02716. One of the authors (VD) is grateful to the A. Mickiewicz University at Poznań for hospitality. 


\section{REFERENCES}

[1] Aliev F. G., Kunnen E., Temst K., Mae K., Verbanck G., Barnaś J., Moshchalkov V. V. and Bruynseraede Y., Phys. Rev. Lett., 78 (1997) 134.

[2] Simonds J. L., Phys. Today 48 (1995) 26.

[3] Kools J. C. S., IEEE Trans. Magn., 32 (1965) 3165.

[4] Gider S., Runge B.-U., Marley A. C. and Parkin S. S. P., Science, 281 (1998) 797.

[5] Altshuler B. L., Aronov A. G., Khmelnitskit D. E. and Larkin A. I., in Quantum Theory of Solids, edited by I. M. Lifshits (Mir, Moscow) 1982, pp. 130-237.

[6] Lee P. A. and Ramakrishnan T. V., Rev. Mod. Phys., 57 (1985) 287.

[7] Altshuler B. L. and Aronov A. G., in Electron-Electron Interaction in Disordered Systems, edited by A. L. Efros and M. Pollak (North-Holland, Amsterdam) 1985.

[8] Kobayashi S., Oоtuka Y., Komori F. and Sasaki W., J. Phys. Soc. Jpn., 51 (1982) 689.

[9] Rubinstein M., Rachford F. J., Fuller W. W. and Prinz G. A., Phys. Rev. B, 37 (1988) 8689.

[10] Aprili M., Lesueur J., Dumoulin L. and Nédellec P., Solid State Commun., 102 (1997) 41.

[11] Singh A. and Fradkin E., Phys. Rev. B, 35 (1987) 6894.

[12] Altshuler B. L. and Aronov A. G., Solid State Commun., 46 (1983) 429.

[13] Hood R. Q., Falicov L. M. and Penn D. R., Phys. Rev. B, 49 (1994) 368.

[14] Barnaś J. and Fert A., Phys. Rev. B, 49 (1994) 12835. 